UNITUE-THEP-07/2002

http://xxx.lanl.gov/abs/hep-ph/0202202

\title{
Infrared Exponents and Running Coupling of SU(N) Yang-Mills Theories
}

\author{
C. S. Fischer ${ }^{1 \dagger}$ and R. Alkofer ${ }^{2}$ \\ Institute for Theoretical Physics, Tübingen University \\ Auf der Morgenstelle 14, D-72076 Tübingen, Germany
}

\begin{abstract}
We present approximate solutions for the gluon and ghost propagators as well as the running coupling in Landau gauge Yang-Mills theories. We solve the corresponding Dyson-Schwinger equations in flat Euclidean space-time without any angular approximation. This supplements recently obtained results employing a four-torus, i.e. a compact space-time manifold, as infrared regulator. We confirm previous findings deduced from an extrapolation with tori of different volumes: the gluon propagator is weakly vanishing in the infrared and the ghost propagator is highly singular. For non-vanishing momenta our propagators are in remarkable agreement with recent lattice calculations.
\end{abstract}

Keywords: Confinement, Non-perturbative QCD, Running coupling constant, Gluon propagator, Dyson-Schwinger equations, Infrared behaviour

PACS: 12.38.Aw 14.70.Dj 12.38.Lg 11.15.Tk 02.30.Rz

\footnotetext{
†Supported by the European Graduate School Basel-Tuebingen.

${ }^{1}$ E-Mail:chfi@axion01.tphys.physik.uni-tuebingen.de

${ }^{2}$ E-Mail:reinhard.alkofer@uni-tuebingen.de
} 
The non-perturbative structure of the gluon propagator is of fundamental interest in Quantum Chromo Dynamics, and its knowledge would provide an important input for many calculations in hadron physics [1]. As infrared singularities might be encountered, a nonperturbative continuum method seems mandatory. In this context, the Landau gauge DysonSchwinger equations have been solved analytically in the infrared, but had to be truncated in order to get a closed system of equations [2, 3, 4, 5, 6]. On the other hand, lattice calculations [7, 8] include all non-perturbative physics of Yang-Mills theories but are limited for small momenta by the finite lattice volume. Despite these shortcomings both approaches agree surprisingly well in their general statements: there is clear evidence for an infrared finite or even vanishing gluon propagator and a diverging ghost propagator. This is in accordance with the Kugo-Ojima confinement criterion, which in Landau gauge includes the statement that the ghost propagator should be more singular than a simple pole [9].

One of the obstacles encountered in extracting definite values of infrared exponents in Dyson-Schwinger studies is the angular integrals inherent to these equations. Therefore approximated treatments of the angular integrals have been applied in numerical works so far [2, 3]. In general the angular approximations proved to be good for high momenta but less trustworthy in the infrared. Recent studies [4, 5, 6] therefore concentrated on the infrared analysis, where exact results have been gained for the limit of vanishing momentum.

In a previous paper [10] a truncation scheme has been presented, which on the one hand provides the correct anomalous dimensions of the ghost and gluon dressing functions, $Z\left(k^{2}\right)$ and $G\left(k^{2}\right)$, in the ultraviolet region of momentum. On the other hand, it reproduces the infrared exponents found in [5, 6] which are close to the ones extacted from lattice calculations [7, 8]. The coupled set of equations for the ghost and gluon dressing functions on a fourtorus have been solved recently [10]. This turned out to be an effective tool to overcome the angular approximation for non-vanishing momenta. The numerical solutions proved to be compatible with only one out of two solutions of the infrared analysis of refs. [5, 6], thus suggesting that not every analytical solution for zero momentum connects to numerical solutions for non-vanishing momenta. However, employing the torus as an infrared regulator shares with lattice calculations the problem of working on a finite volume. Therfore further investigations are highly desirable.

In this letter we present the solution of the coupled set of equations described in ref. [10] in flat Euclidean space-time. The results confirm our previous findings on the torus. We obtain a numerical solution for the gluon and the ghost propagator with infrared behavior $D_{g l}\left(k^{2}\right) \sim\left(k^{2}\right)^{2 \kappa-1}, \kappa \approx 0.595$ and $D_{g h}\left(k^{2}\right) \sim\left(k^{2}\right)^{-\kappa-1}$. Correspondingly we find an infrared 


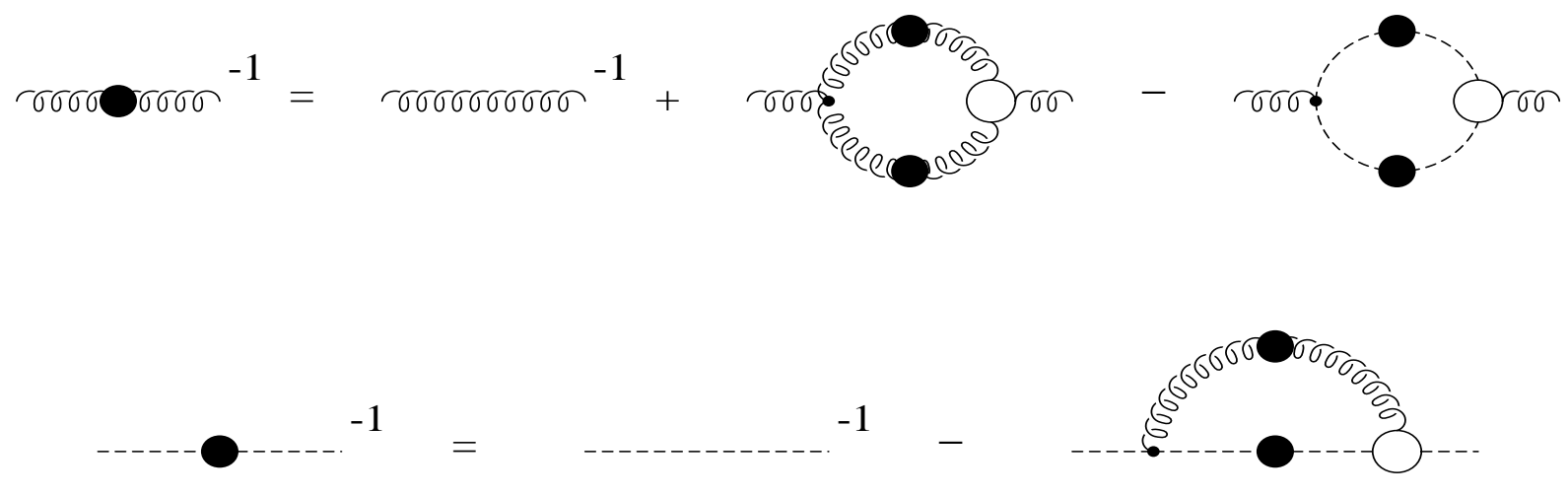

Figure 1: Diagrammatic representation of the truncated gluon and ghost Dyson-Schwinger equations studied in this letter. Terms with four-gluon vertices have been dismissed.

fixed point of the running coupling, $\alpha(0) \approx 2.972$ for the gauge group $\mathrm{SU}(3)$.

\section{The truncation scheme}

To make this letter self-contained we will give a short summary of the truncation scheme introduced in detail in ref. [10]. It has turned out in previous studies [6], that the dressing of the three-point vertex functions does not alter the qualitative behaviour of the ghost and gluon system. We therefore restrict ourselves to bare vertices for simplicity. Furthermore all diagrams including four-gluon vertices have been dropped. A diagrammatical representation of the resulting system of equations is presented in Fig. 11. To extract the gluon dressing function the gluon equation has to be contracted with a tensor. Maintaining Lorentz invariance its general form reads

$$
\mathcal{P}_{\mu \nu}^{(\zeta)}(k)=\delta_{\mu \nu}-\zeta \frac{k_{\mu} k_{\nu}}{k^{2}} .
$$

The parameter $\zeta$ allows to continuously interpolate from the transversal projector, $\zeta=1$, to the Brown-Pennington projector [1], $\zeta=4$. In a full treatment without truncation the gluon equation would be transverse and the propagators would not depend on $\zeta$. We will see later on to what extent transversality is violated in the present truncation scheme.

Closely connected to the subject of transversality is the appearance of unphysical quadratic divergencies in the gluon equation. For $\zeta=4$ the equation is projected on the longitudinal tensor $k_{\mu} k_{\nu} / k^{2}$ and no quadratic ultraviolet divergences appear [11]. For a transverse polarization the gluon equation would be independent of $\zeta$, and thus no quadratic divergences would occur for all $\zeta$ in a full equation. In the practical case, the loss of transversality causes quadratic divergences to appear and their careful removal is essential for a numerical solution of the system. However, this procedure should not change the infrared properties 
of the equations. Anticipating that the ghost loop is the leading contribution to the infrared we subtract the quadratically ultraviolet divergent constant in the gluon loop.

The resulting system of equations for the ghost dressing function $G(x)$ and the gluon dressing function $Z(x)$ reads

$$
\begin{aligned}
& \frac{1}{G(x)}=\tilde{Z}_{3}-g^{2} N_{c} \int \frac{d^{4} q}{(2 \pi)^{4}} \frac{K(x, y, z)}{x y} G(y) Z(z) \\
& \frac{1}{Z(x)}=Z_{3}+g^{2} \frac{N_{c}}{3} \int \frac{d^{4} q}{(2 \pi)^{4}} \frac{M(x, y, z)}{x y} G(y) G(z)+Z_{1} g^{2} \frac{N_{c}}{3} \int \frac{d^{4} q}{(2 \pi)^{4}} \frac{Q(x, y, z)}{x y} Z(y) Z(z) .
\end{aligned}
$$

The symbols $x:=k^{2}$ and $y:=q^{2}$ denote squared momenta where $z=x+y-2 \sqrt{x y} \cos \Theta$. The kernels ordered with respect to powers of $z$ have the form:

$$
\begin{aligned}
K(x, y, z)= & \frac{1}{z^{2}}\left(-\frac{(x-y)^{2}}{4}\right)+\frac{1}{z}\left(\frac{x+y}{2}\right)-\frac{1}{4} \\
M(x, y, z)= & \frac{1}{z}\left(\frac{\zeta-2}{4} x+\frac{y}{2}-\frac{\zeta}{4} \frac{y^{2}}{x}\right)+\frac{1}{2}+\frac{\zeta}{2} \frac{y}{x}-\frac{\zeta}{4} \frac{z}{x}, \\
Q(x, y, z)= & \frac{1}{z^{2}}\left(\frac{1}{8} \frac{x^{3}}{y}+x^{2}-\frac{19-\zeta}{8} x y+\frac{5-\zeta}{4} y^{2}+\frac{\zeta}{8} \frac{y^{3}}{x}\right) \\
& +\frac{1}{z}\left(\frac{x^{2}}{y}-\frac{15+\zeta}{4} x-\frac{17-\zeta}{4} y+\zeta \frac{y^{2}}{x}\right) \\
& -\left(\frac{19-\zeta}{8} \frac{x}{y}+\frac{17-\zeta}{4}+\frac{9 \zeta}{4} \frac{y}{x}\right) \\
& +z\left(\frac{\zeta}{x}+\frac{5-\zeta}{4 y}\right)+z^{2} \frac{\zeta}{8 x y}+\frac{5}{4}(4-\zeta) .
\end{aligned}
$$

Here the last term in the gluon kernel $Q$ is introduced by hand to subtract all quadratic divergencies from the ghost and gluon loop, see ref. [10].

In order to ensure the correct one loop scaling of the dressing functions in the ultraviolet within this truncation scheme one has to violate the Slavnov-Taylor identity $Z_{1}=Z_{3} / \tilde{Z}_{3}$, similar to the treatment in [2]. Refering the reader to the detailed derivation in [10], we only give the resulting momentum dependent substitution $\mathcal{Z}_{1}$ for $Z_{1}$ :

$$
Z_{1} \rightarrow \mathcal{Z}_{1}(x, y, z)=\frac{G(y)^{(1-a / \delta-2 a)}}{Z(y)^{(1+a)}} \frac{G(z)^{(1-b / \delta-2 b)}}{Z(z)^{(1+b)}}
$$

With this substitution the logarithmic behaviour of the gluon loop in the ultraviolet is the same as the one of the ghost loop. The coefficients in the kernels then sum up to the correct anomalous dimensions, namely $\delta=-9 / 44$ for the ghost and $\gamma=-13 / 22$ for the gluon propagator. The parameters $a$ and $b$ can be chosen freely. In our calculations we use $a=b=3 \delta$, which has been shown 10 to minimize the momentum dependence of $\mathcal{Z}_{1}$. Note 
that $a=b=0$ leads to the truncation scheme of ref. 12 and $a=3 \delta, b=0$ together with appropriate vertex dressings to the one of ref. [2].

\section{Infrared analysis}

In the truncation schemes of refs. [2, 3, 4, 5, 6, 10] the ghost loop provides the infrared leading term in the gluon equation. The only exception is given in ref. [12]: in this scheme also the gluon loop contributes to the same order as the ghost loop. As we do not want to change the infrared behaviour by manipulations of the renormalization we choose $a=b=3 \delta$ which is the only choice to give a constant $\mathcal{Z}_{1}$ in the infrared. Then the gluon loop does not contribute to the infrared behaviour of the equations. Therefore the leading analytical solutions found in the ghost loop only truncations of [4] with $\zeta=4$ and of [5, 6] with $\zeta=1$, are found in our case as well [10]. In the following we will determine subleading contributions in the infrared, and judge their importance for the numerical treatment of the coupled system of equations.

We employ the ansatz

$$
\begin{aligned}
& Z(x)=A x^{2 \kappa}\left(1+f x^{\rho}\right) \\
& G(x)=B x^{-\kappa}\left(1+g x^{\rho}\right)
\end{aligned}
$$

in the eqs. (2) and (3). The left hand side and the integrands are expanded to appropriate powers of momentum. After integration (for technical details see refs. [6, 10, 13]) the coefficients of equal powers on both sides of the equations have to match for consistency. The conditions on the leading term remain unchanged. Hence for an arbitary projector the leading power $\kappa$ is given by

$$
\frac{(2+\kappa)(1+\kappa)}{18(3-2 \kappa)}-\frac{4 \kappa-2}{4 \zeta \kappa-4 \kappa+6-3 \zeta}=0 .
$$

This equation has at least two solutions for a given $\zeta$ from which only one connects to the numerical solution for finite momenta [10]. The result for the running coupling is given by

$$
\alpha(0)=\frac{4 \pi}{18} \frac{\Gamma(3-2 \kappa) \Gamma(3+\kappa) \Gamma(1+\kappa)}{\Gamma^{2}(2-\kappa) \Gamma(2 \kappa)} \approx 2.972
$$

where the nonperturbative definition $\alpha(x)=\alpha\left(\mu^{2}\right) Z(x) G^{2}(x)$ has been used.

Matching subleading powers leads to the coupled set of homogeneous equations for $f, g$ 
and $\rho$ :

$$
\begin{aligned}
& \left(3 \nu \frac{6 \kappa(\kappa-1)(-3+2 \kappa)}{(\kappa+\rho-2)(\kappa+\rho-1)(\kappa+\rho)} \frac{\Gamma(2 \kappa) \Gamma(2-\kappa+\rho) \Gamma(3-\kappa-\rho)}{\Gamma(4-2 \kappa) \Gamma(1+\kappa-\rho) \Gamma(3+\kappa+\rho)}-1\right) g \\
& +\left(3 \nu \frac{3(-2+2 \kappa+\rho)}{2(\kappa+\rho-2)(\kappa+\rho-1)(\kappa+\rho)} \frac{\Gamma(2-\kappa) \Gamma(2 \kappa+\rho+1) \Gamma(3-\kappa-\rho)}{\Gamma(3-2 \kappa-\rho) \Gamma(1+\kappa) \Gamma(3+\kappa+\rho)}\right) f=0 \\
& \left(\nu \frac{4 \zeta \kappa-4 \kappa+2 \rho-2 \zeta \rho+6-3 \alpha}{2 \kappa-\rho-1} \frac{\Gamma(2-\kappa) \Gamma(2 \kappa-\rho) \Gamma(2-\kappa+\rho)}{\Gamma(1+\kappa) \Gamma(1+\kappa-\rho) \Gamma(4-2 \kappa+\rho)}\right) g+f=0 .
\end{aligned}
$$

Here $\nu=N_{c} g^{2} A B^{2} / 48 \pi^{2}=\alpha(0) / 4 \pi$. There is either the trivial solution $f=g=0$ or one has to set the determinant of these linear equations to zero. We then obtain the results $\rho_{(1)}=0, \rho_{(2)}=0.58377, \rho_{(3)}=1.20300$ and several other solutions with higher values of the power. The solution $\rho_{(1)}=0$ corresponds to the pure power solution. The lowest nonvanishing solution, $\rho_{(2)}=0.58377$, is sufficently high that we safely may neglect it in the numerical treatment of the infrared part of the equations. This will be detailed in the next section.

\section{Numerical Method and Results}

Two of the four integrals in equations (2) and (3) can be done trivially and yield a factor of $4 \pi$, while the remaining angular integral and the radial integral have to be performed with the help of numerical routines. To achieve high accuracy we split the radial loop integral into three parts, $y \in\left[0, \epsilon^{2}\right], y \in\left(\epsilon^{2}, x\right]$ and $y \in\left(x, x_{U V}\right]$. The integrals will be computed numerically, however according to the value of their argument the dressing functions $Z$ and $G$ have to be handled differently. In the infrared region, $y, z \in\left[0, \epsilon^{2}\right], Z$ and $G$ behave like powers and are replaced according to equations (8) with $f=g=0$. The infrared matching point is chosen to be $\epsilon^{2}=(0.55 \mathrm{MeV})^{2}$ in our calculations. In the high momentum regime, $y \in\left(x, x_{U V}\right]$, arguments $z$ occur which are larger than the numerical cutoff $x_{U V}$. There we approximate the respective dressing functions by the expressions

$$
\begin{aligned}
& Z(z)=Z(s)\left[\omega \log \left(\frac{z}{s}\right)+1\right]^{\gamma}, \\
& G(z)=G(s)\left[\omega \log \left(\frac{z}{s}\right)+1\right]^{\delta} .
\end{aligned}
$$

according to the one loop behaviour of the solutions as has been detailed in [10]. Here $\omega=$ $11 N_{c} \alpha(s) / 12 \pi$ and the squared momentum $s$ is a perturbative scale. We chose $s=(174 \mathrm{GeV})^{2}$ to be slightly lower than the numerical cutoff $x_{U V}=(177 \mathrm{GeV})^{2}$. To be able to perform the angular integrations for momenta $\left[\epsilon^{2}, x_{U V}\right]$ we expand the dressing functions in Chebychev polynomials and solve the coupled system of equations for the expansion coefficients using a Newton iteration method. Details of this technique can be found in ref. [3]. 
$\mathrm{Z}(\mathrm{x})$

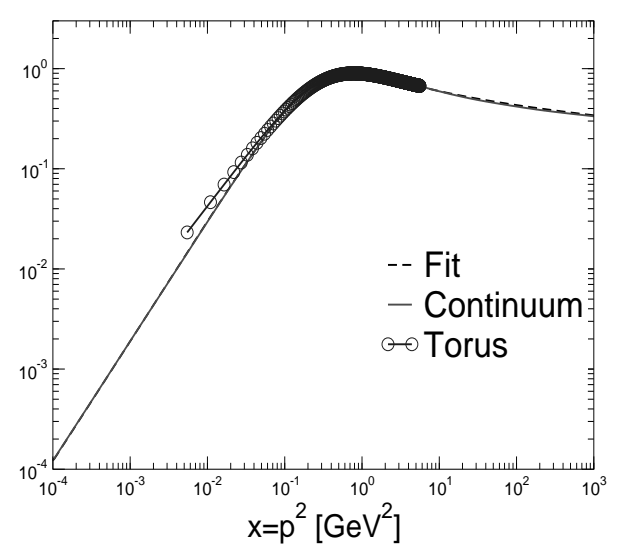

$G(x)$

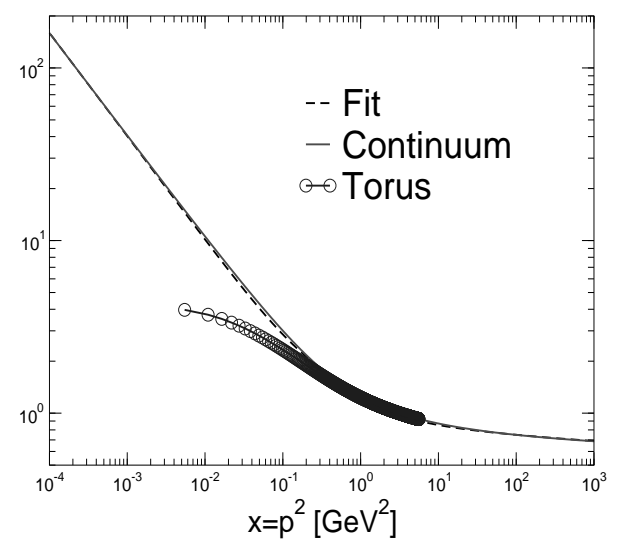

$\alpha(x)$

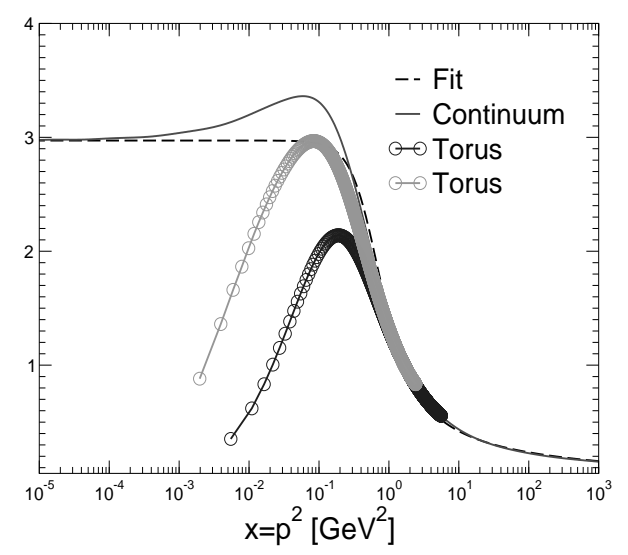

Figure 2: Shown are the results for the gluon dressing function, the ghost dressing function and the running coupling using a transverse projector. The solutions are compared to previous results on a torus, for the running coupling two results for two different volumes are presented. The dashed lines are the fits given in eq. (14).

We apply a MOM regularization scheme similar to the ones used previously in refs. [2, 3]. For numerical reasons it is favourable to subtract the ghost equation at zero momentum and the gluon equation at the scale $s$ introduced above. Due to this the unknown renormalization constants $Z_{3}$ and $\tilde{Z}_{3}$ drop out and instead of them one has to specify two input variables. We chose the infrared parameter $A$ (c.f. eq. (8)), and implement the condition $Z\left(\mu^{2}\right) G^{2}\left(\mu^{2}\right)=1$. Furthermore, one has to specify the coupling $\alpha\left(\mu^{2}\right)=g^{2} / 16 \pi^{2}$ entering eqs. (2, (3)). Finally we have fixed the momentum scale by calculating the running coupling for the colour group $\mathrm{SU}(3)$, and requiring the experimental value $\alpha(x)=0.118$ at $x=M_{Z}^{2}=(91.187 \mathrm{GeV})^{2}$ [14].

In Fig. 2 we show the results for the transverse projector, $\zeta=1$. The gluon and ghost dressing functions behave powerlike for low momenta and obey one loop scaling in the ultraviolet as expected. Accordingly the running coupling has a fixed point in the infared 

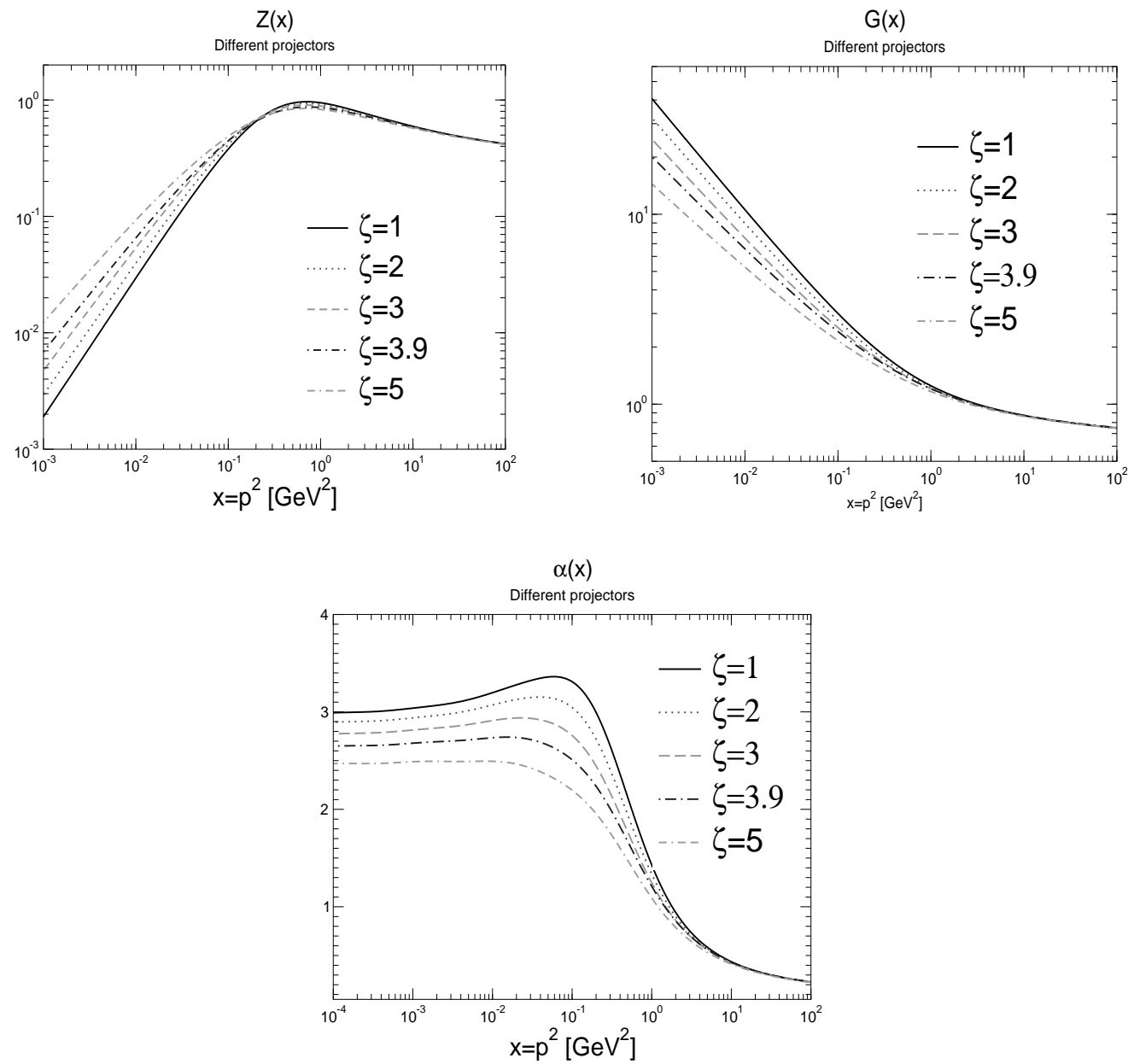

Figure 3: Shown are the results for the gluon dressing function, the ghost dressing function and the running coupling, c.f. fig. 2, for different projectors.

and decreases logarithmically in the perturbative regime above several GeV. For intermediate momenta the behaviour of the running coupling seems to suggest a second zero of the $\beta$ function around $(100 \mathrm{MeV})^{2}$. However, such an extremum in $\alpha(x)$ would result in a double valued $\beta$-function, therefore we regard it as an artefact of our truncation scheme. In fact with subleading gluon contributions in the infrared and perturbative suppression of the two loop contributions in the ultraviolet it is exactly the intermediate momentum regime where the omission of the two loop diagrams should cause deficiencies in our solutions.

The asymptotic behaviour of the solutions can also be seen from the functional form of our fits. The dressing functions $Z(x)$ and $G(x)$ can be described by

$$
\begin{array}{rlrl}
\alpha(x)=\frac{\alpha(0)}{\ln \left(e+a_{1} x^{a_{2}}+b_{1} x^{b_{2}}\right)}, & R(x) & =\frac{c x^{\kappa}+d x^{2 \kappa}}{1+c x^{\kappa}+d x^{2 \kappa}}, \\
Z(x)=\left(\frac{\alpha(x)}{\alpha(\mu)}\right)^{1+2 \delta} R^{2}(x), & G(x)=\left(\frac{\alpha(x)}{\alpha(\mu)}\right)^{-\delta} R^{-1}(x),
\end{array}
$$


with fitting parameters $a_{1}, a_{2}, b_{1}, b_{2}$ and $c, d$ for the running coupling $\alpha(x)$ and the auxiliary function $R(x)$ respectively. The values of $\alpha(0)=2.972$ and $\kappa=0.5953$ are known from the infrared analysis in the previous paragraph, whereas $\delta=-9 / 44$ is the anomalous dimension of the ghost dressing function and $\alpha\left(\mu^{2}\right)=0.9676$. The six parameters of the fit are given by $a_{1}=5.292 \mathrm{GeV}^{-2 a_{2}}, a_{2}=2.324, b_{1}=0.034 \mathrm{GeV}^{-2 b_{2}}, b_{2}=3.169, c=1.8934 \mathrm{GeV}^{-2 \kappa}$ and $d=4.6944 \mathrm{GeV}^{-4 \kappa}$. As can be seen in Fig. 2 the fit works very well and can be used as input for phenomenological calculations in future work.

Our results for different values of the parameter $\zeta$ (c.f. eq. (11)) are shown in Fig. 3. In accordance with the infrared analysis the power $\kappa$ changes from $\kappa=0.5953$ for $\zeta=1$ to $\kappa=0.4610$ for $\zeta=5$. The perturbative properties of the solutions remain unchanged. The bump in the running coupling gets smaller but does not disappear even for $\zeta=5$. It has already been stated above that the dressing functions would be independent of $\zeta$ in a complete treatment of the gluon equation. As all our solutions are very similar even on a quantitative level we conclude that transversality is lost only to a moderate extent. This is a somewhat surprising result in such a simple truncation scheme as the one at hand.

The Brown-Pennington projector, $\zeta=4$, is an exceptional case as can be seen from eq. (9). Here the $\kappa$-dependence of the second term cancels and only one solution, $\kappa=1$, can be found (c.f. ref. [4]). We found no numerical solutions for this case. However, within the limit of numerical accuracy, solutions for $\zeta$ slightly different from 4 can be found leading to a value for $\kappa$ slightly different from $1 / 2$. E.g. in Fig. 3 the case $\zeta=3.9$ leading to $\kappa=0.5038$ is depicted.

Finally, we compare our results to recent lattice calculations for two colours [15]. As the solutions on the lattice include all non-perturbative effects, the results shown in Fig. 1 confirm again that the omission of the two loop diagrams in our truncation scheme mostly effects the region around the bending point at $1 \mathrm{GeV}$. However, in general the qualitative and partly even quantitative agreement of the two methods is remarkable. The combined evidence of the two methods points strongly towards an infrared vanishing gluon propagator and an infrared singular ghost propagator in Landau gauge.

\section{Conclusions}

In this letter we have presented approximate non-perturbative solutions for the gluon and the ghost propagators as well as the running coupling in Landau gauge. We obtained these solutions for the Dyson-Schwinger equations in the truncation of ref. [10], working with bare vertices and omitting all diagrams that involve four-gluon vertices. An improvement 
DSE vs. lattice results $\left(16^{3} \times 32\right)$

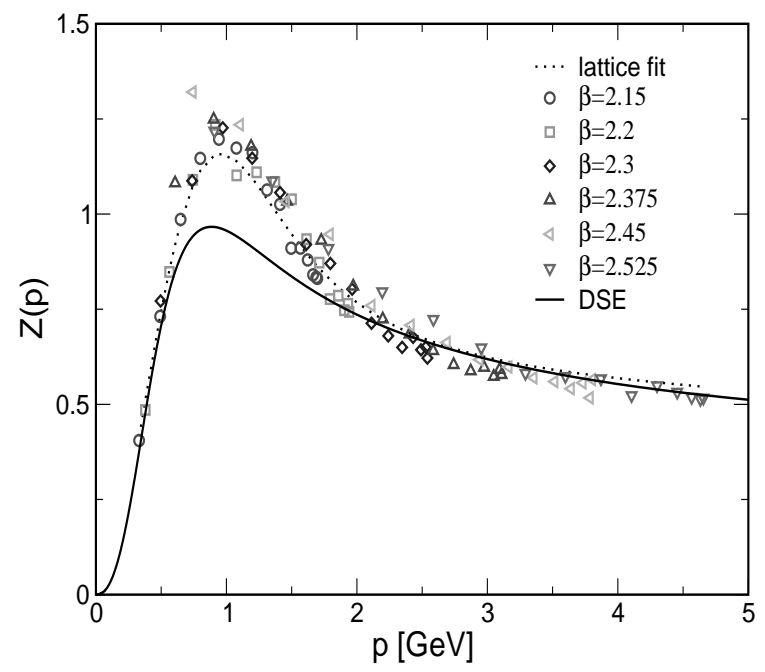

DSE vs. lattice results $\left(16^{3} \times 32\right)$

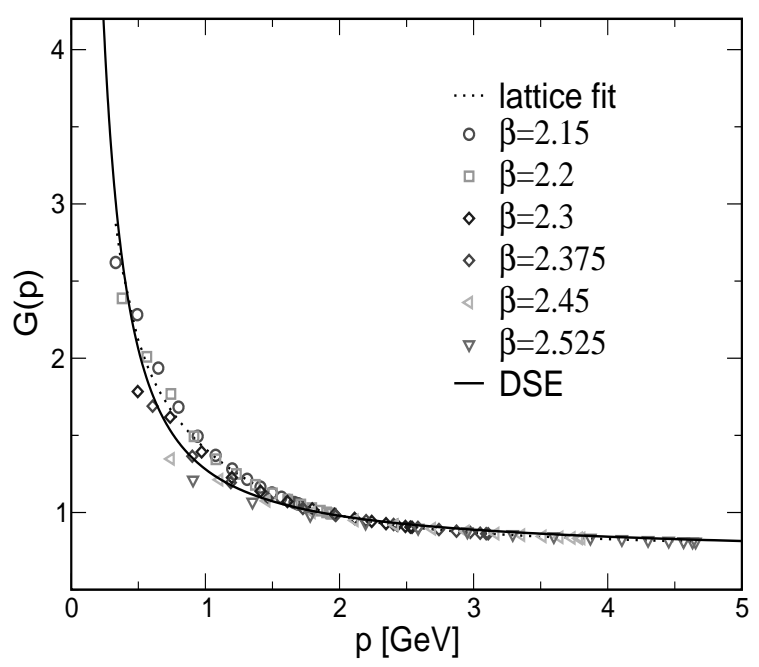

Figure 4: Solutions of the Dyson-Schwinger equations compared to recent lattice results for two colours [15].

to previous treatments has been the explicit numerical calculation of all angular integrals thus overcoming the angular approximations that have been made so far. It is interesting to note that only one solution (for a given projector) has been found. Furthermore, we could demonstrate that our truncation scheme violates transversality of the gluon equation only to a moderate extent. Previous findings on a four-torus have been checked and found to be in agreement with the flat space-time solutions up to effects of finite volume in the very infrared. Despite the simplicity of the truncation our solutions agree remarkably well with recent lattice calculations. For the infrared behaviour of Landau gauge Green's functions circumstancial evidence points towards a weakly infrared vanishing or even finite gluon propagator, while the ghost propagator seems to rise more strongly than a simple pole.

\section{Acknowledgements}

We thank Jacques Bloch, Hugo Reinhardt, Sebastian Schmidt, Peter Watson and Daniel Zwanziger for helpful discussions. We are especially grateful to Lorenz von Smekal for many valuable discussions and for making ref. [6] available to us. We are indebted to Kurt Langfeld for communicating and elucidating his lattice results, partly prior to publication.

This work has been supported by the DFG under contract Al 279/3-3 and by the European graduate school Tübingen-Basel. 


\section{References}

[1] R. Alkofer and L. von Smekal, Phys. Rept. 353 (2001) 281 arXiv:hep-ph/0007355. C. D. Roberts and S. M. Schmidt, Prog. Part. Nucl. Phys. 45 (2000) S1 arXiv:nucl-th/0005064.

[2] L. von Smekal, R. Alkofer and A. Hauck, Phys. Rev. Lett. 79 (1997) 3591 arXiv:hepph/9705242]; Annals Phys. 267 (1998) 1 arXiv:hep-ph/9707327.

[3] D. Atkinson and J. C. Bloch, Phys. Rev. D58 (1998) 094036 arXiv:hep-ph/9712459.

[4] D. Atkinson and J.C. Bloch, Mod. Phys. Lett. A13 (1998) 1055 arXiv:hep-ph/9802239.

[5] D. Zwanziger, arXiv:hep-th/0109224.

[6] C. Lerche and L. von Smekal, arXiv:hep-ph/0202194;

C. Lerche, Diploma Thesis, Erlangen University, June 2001 (in German).

[7] F. D. Bonnet, P. O. Bowman, D. B. Leinweber and A. G. Williams, Phys. Rev. D 62 (2000) 051501 arXiv:hep-lat/0002020;

F. D. Bonnet et al., Phys. Rev. D 64(2001) 034501 [arXiv:hep-lat/0101013].

[8] K. Langfeld, H. Reinhardt and J. Gattnar, Nucl. Phys. B 621 (2002) 131 arXiv:hepph/0107141; arXiv:hep-lat/0110025.

[9] T. Kugo, Int. Symp. on BRS symmetry, Kyoto, Sep. 18-22, 1995, arXiv:hep-th/9511033, see also:

P. Watson and R. Alkofer, Phys. Rev. Lett. 86 (2001) 5239 arXiv:hep-ph/0102332;

R. Alkofer, L. von Smekal and P. Watson, Proceedings of the ECT* Collaboration Meeting on Dynamical Aspects of the QCD Phase Transition, Trento, Italy, March 12-15, 2001, arXiv:hep-ph/0105142.

[10] C. S. Fischer, R. Alkofer and H. Reinhardt, arXiv:hep-ph/0202195.

[11] N. Brown and M. R. Pennington, Phys. Rev. D 38 (1988) 2266.

[12] J. C. Bloch, Phys. Rev. D 64 (2001) 116011 arXiv:hep-ph/0106031.

[13] A. Hauck, L. von Smekal and R. Alkofer, Comput. Phys. Commun. 112 (1998) 149 arXiv:hep-ph/9604430; ibid 166 arXiv:hep-ph/9804376.

[14] D.E. Groom et al. (Particle Data Group), Eur. Phys. J. C15, (2000) 1, and 2001 partial update for edition 2002 (URL: http://pdg.lbl.gov).

[15] K. Langfeld, private communication. 